\title{
La pharmacie hospitalière à Séville au XVIIle siècle
}

Maria Teresa López Diaz, Consolación Martinez Garcia, Esteban Moreno Toral

\section{Citer ce document / Cite this document :}

López Diaz Maria Teresa, Martinez Garcia Consolación, Moreno Toral Esteban. La pharmacie hospitalière à Séville au XVIIle siècle. In: Revue d'histoire de la pharmacie, $84^{\mathrm{e}}$ année, $\mathrm{n}^{\circ} 312,1996$. Actes du XXXle Congrès International d'Histoire de la Pharmacie (Paris, 25-29 septembre 1995) pp. 66-76.

doi : $10.3406 /$ pharm.1996.6160

http://www.persee.fr/doc/pharm_0035-2349_1996_num_84_312_6160

Document généré le 16/10/2015 


\title{
La pharmacie hospitalière à Séville au XVIII ${ }^{\mathrm{e}}$ siècle
}

\author{
M. T. LOPEZ DIAZ ; C. MARTINEZ GARCIA ; E. MORENO TORAL \\ Seville, ESPAGNE
}

\section{Introduction}

Pendant le XVIII ${ }^{\mathrm{e}}$ siècle la ville de Séville comptait un important nombre d'hôpitaux, dix-huit au total, qui présentaient diverses caractéristiques et finalités (1) les hôpitaux fondés par les Confréries de Charité, dédiés les uns à l'exercice de la bienfaisance, les autres à une action sociale ; les orphelinats et asiles, à soigner et protéger les orphelins et les personnes âgées, respectivement ; et les thérapeutiques, qui au point de vue sanitaire sont les seuls que nous pouvons considérer comme véritables hôpitaux, car bien qu'ils étaient également des institutions d'aide de type charitable, nous pouvons considérer qu'ils avaient une organisation médicale et qu'ils dispensaient à la population une assistance médico-sanitaire.

Les hôpitaux de soins thérapeutiques étaient dix au total, et dans chacun d'eux on traitait une maladie déterminée : Hôpital del Amor de Dios (1587-1837) (2), dédié aux soins de toute sorte de fièvres et maladies aiguës, chroniques, épidémiques et saisonnières; Hôpital del Espíritu Santo (1587-1837) (3), originairement de Santa Catalina de los Desamparados, pour les malades porteurs de bubons, plaies et autres douleurs dérivées de cette maladie. Dans ces deux centres furent fusionnés 76 des hôpi- taux réduits en 1586; Hôpital de San Hermenegildo ou du Cardenal (14531837) (4), pour la guérison d'hommes blessés par des armes à feu, poignards, brûlures de bras et de jambes, d'os, foulures et toutes autres blessures n'étant pas arrivé au stade de plaies ayant besoin de mercuriels ; Hôpital de San Lázaro (1249) (5), pour la guérison des malades de la lèpre (diformes, envahis de plaies et lépreux); Hôpital de San Cosme y San Damián, vulgairement des Inocentes (1436?1840) (6), dédié aux soins des déments ; Hôpital de la Santa Caridad (1664), pour les malades incurables qui ne furent pas admis dans d'autres hôpitaux et pour des malades phtisiques; Hôpital de las Cinco Llagas o de la Sangre (1500) pour les hommes et femmes affectés de toute maladie que ce soit non contagieuse ; Hôpital de San Cosme y San Damián ou de las Bubas (1383-1837), pour le traitement des malades affectés de cette maladie ; Hôpital de Nuestra Sra. de los Desamparados (1698-1837)(7), pour les malades touchés par la tuberculose pulmonaire; Hôpital de Nuestra Sra. de la Paz (1574), originairement de la Cruz ou de las Tablas, pour les hommes incurables. Tous ces hôpitaux étaient de patronage ecclésiastique à l'exception de l'Hôpital de las Bubas dont le patronage appartenait au Conseil municipal de la ville de Séville. 
La plupart d'entre eux cessèrent de fonctionner en 1837, année durant laquelle eut lieu leur centralisation dans l'Hôpital de las Cinco Llagas, qui alors s'appela également Hôpital Central.

De ces dix centres hospitaliers seuls cinq d'entre eux eurent depuis leur fondation une apothicairerie H. del Amor de Dios, H. del Espíritu Santo, H. del Cardenal, H. de Las Cinco Llagas et $\mathrm{H}$. de las Bubas - qu'ils maintinrent tant bien que mal jusqu'à leur centralisation. L'existence de ces apothicaireries et tout ce qui est en relation avec le service pharmaceutique fut réglementé par les Constitutions et Ordonnances établissant l'organisation et la direction des hôpitaux ci-dessus, lesquelles restèrent en vigueur, avec à peine quelques changements, durant tout le XVIII ${ }^{\mathrm{e}}$ siècle.

En fonction de ce qui était établi dans ces dernières et des renseignements provenant de la documentation appartenant à ce siècle en relation avec l'organisation sanitaire de ces centres, nous avons mené à terme une synthèse générale sur l'assistance pharmaceutique dans ces hôpitaux de Séville durant le XVIII ${ }^{\mathrm{e}}$ siècle. Après avoir regroupé tous les renseignements ci-dessus mentionnés et ayant fait une étude comparative de ces derniers, nous pouvons affirmer que l'organisation des apothicaireries des hôpitaux - fonctions et attributions, droits et devoirs qui leur étaient imposés - s'établit avec les mêmes prémisses.

\section{Installation et ustensiles}

Toutes les apothicaireries étudiées étaient installées au rez-dechaussée du bâtiment, à côté d'une cour ou jardin avec un puits, où l'on cultivait probablement quelques plan- tes médicinales. Dans la plupart des cas elles se trouvent près des infirmeries. Ces apothicaireries comptent normalement trois dépendances : l'officine de l'apothicairerie proprement dite, où l'on distribuait les médicaments ; l'arrière-boutique, où se trouvaient les appartements de l'apothicaire et des garçons d'apothicairerie; et l'atelier, lieu où l'on confectionnait les médicaments. Dans l'Hôpital del Cardenal on mentionne aussi une espèce de cuisine, " hornillería ", où se trouvaient les fourneaux avec les alambics et peut-être quelqu'autre instrument tel que la presse (8).

Ces apothicaireries sont dans tous les cas très spacieuses, comme on peut le constater d'après les listes d'achats, où les herbes, racines, plantes et fleurs étaient d'habitude acquises en gros, surtout avant l'arrivée du printemps, en les comptabilisant par charges, sacs, arobes et paniers. Ceci est confirmé, également, par les inventaires existants en relation avec ces apothicaireries où l'on peut voir le nombre élevé d'outillage, bocaux et autres ustensiles qu'elles possédaient. A part quelques différences, toutes les apothicaireries ont le matériel suivant: une grande presse avec ses couffins et vis respectifs pour extraire les jus dans le cas de l'Hôpital del Amor de Dios et de l'Hôpital del Espíritu Santo, l'apothicairerie possédait deux presses - ; " alquitaras " ou alambics d'argile ou de verre, pour distiller les racines, feuilles, fleurs et fruits ; des grandes cruches vidriées, pour conserver les différentes eaux distillées. Dans l'Hôpital del Cardenal on indique à ce sujet que l'apothicaire devait disposer de " una pieça señalada para esto. dos hornillos donde se saquen las aguas con ocho alquítaras y comiénzase a destilar desde Abril adelante "; les grands mortiers en fer pour piler les racines, et 
ceux en bronze ou égrugeoirs grands, moyens et petits; des grands fourneaux en fer - l'apothicairerie de l'Hôpital Espíritu Santo en avait deux - ; des poids et des jeux de mesures; des chaudrons en cuivre; écumoirs en fer et en métal; casseroles et cocottes en cuivre; poêles en argile; cuvettes à eau; entonnoirs en verre et en ferblanc; marmites; louches; terrines émaillées; cruches pour la farine et pour le miel, jarres, brocs, fioles, poires en verre, dont le nombre surpasse la cinquantaine dans toutes les apothicaireries étudiées, ainsi que les flacons pour les cordiaux et les sirops, les boîtes en bois et en fer-blanc pour les herbes et les flacons en argile, ces derniers dépassant la centaine.

\section{Approvisionnement et fournisseurs}

Les apothicaireries hospitalières avaient l'habitude de s'approvisionner périodiquement en drogues et médicaments dont elles avaient besoin dans les apothicaireries et dépôts de drogues et d'articles médicinaux, existant dans la ville.

Les apothicaireries ou apothicaires installés à Séville et fournisseurs des hôpitaux de ce siècle furent : Juan Navarro, qui approvisionna l'Hôpital Amor de Dios et l'Hôpital de Las Cinco Llagas ; Francisco de Spino y Guzmán, approvisionna l'Hôpital de Las Cinco Llagas; José Bruno, installé à Sta. María la Blanca, approvisionna l'Hôpital de Las Cinco Llagas; José de Espinosa y Carcel, installé dans la paroisse de Sta. María la Mayor, fournisseur de l'Hôpital Espíritu Santo; Cristobal de Vega, installé dans la rue Francos, qui approvisionna l'Hôpital del Amor de Dios et l'Hôpital de las Cinco Llagas; Salvador Vega, fils du précédent, installé rue Francos, où, en plus de son apothicairerie il possédait depuis au moins l'année 1729, un dépôt de drogues et médicaments qui approvisionnait d'autres apothicaireries publiques ou privées autant en médicaments simples que composés. Le dépôt de Salvador Vega suppose, en tout cas au début, le commencement dans notre ville de ce que l'on appelle "distribution pharmaceutique ». Un autre important dépôt dc drogues fut celui de Juan Antonio Lommaert, auquel à partir de 1750 s'approvisionnaient les apothicaireries des hôpitaux del Amor de Dios, del Cardenal et de las Cinco Llagas.

Dans la deuxième moitié du $\mathrm{XVIII}^{\mathrm{e}}$ siècle il existait à Séville trois autres dépôts de drogues, bien qu'il n'est pas prouvé qu'ils aient approvisionné les hôpitaux : celui des apothicaires Pedro et Manuel Aznar, dans la paroisse de San Esteban; celui de D. José Correa y Amores, dans la paroisse de Sta. María la Mayor; et celui de D. Francisco García Campos, dans la paroisse del Salvador (9). Entre les fournisseurs de l'Hôpital del Espíritu Santo on trouve deux dépôts de drogues installés à Cadiz dans la deuxième moitié du XVIII ${ }^{\mathrm{e}}$ siècle : Raymond y Compañía, et Alonso Martos y Compañía. La plus grande partie des drogues qu'ils fournirent à l'hôpital provenait d'Orient et d'Outre-mer, comme l'opium, l'aloès de Socotora, la racine de Chine, la ronce de Honduras, le jalap, la rhubarbe blanche... et, de ce fait, faciles à trouver dans une ville aussi commerciale et pourvue de nombreuses communications, qu'était Cadix surtout au $\mathrm{XVIII}^{\mathrm{e}}$ siècle, alors qu'elle se trouvait dans toute sa splendeur puisqu'elle était le port d'attache des flottes et galions des Indes (10). D'autres fournisseurs assidus des hôpitaux sont les herboristes, qui très souvent fournissent des plantes médi- 
cinales. Pour les autres produits pas vraiment pharmaceutiques - le miel, l'huile, l'eau-de-vie, le sucre, etc. nécessaires pour la fabrication de certains médicaments, l'apothicairerie hospitalière s'approvisionnait habituellement à l'office de l'hôpital luimême.

\section{Inspections}

La qualité des médicaments et des drogues et en général le bon fonctionnement de l'apothicairerie et du travail de l'apothicaire étaient contrôlés par des inspections périodiques. Les inspections ou visites auxquelles étaient sujet les apothicaireries hospitalières étaient de trois sortes : les visites générales organisées par les Directeurs de l'hôpital; la visite que réalisait l'administrateur de l'hôpital accompagné du médecin et du chirurgien du centre ; et celle réalisée par les visiteurs commissionnés par le Royal Protomedicato.

Les visites ou inspections réalisées par les directeurs et par l'administrateur étaient dans tous les cas enregistrées dans les Constitutions des hôpitaux. Les visites générales réalisées dans les hôpitaux dépendant de la Hiérarchie Ecclésiastique, étaient effectuées par l'Archevêque en personne ou par son Vicaire. Dans le cas de l'Hôpital de las Bubas, dont la direction incombait à la ville, les personnes chargées d'effectuer ces visites étaient les membres du Conseil Municipal, deux des vingt-quatre gentilhommes conseillers (les gentilhommes vingt-quatre) nommés à cet effet par tirage au sort. Lors de ces visites générales les directeurs vérifiaient le bon fonctionnement du centre et l'accomplissement de ce qui avait été établi dans les Constitutions.
Dans les Constitutions de l'Hôpital del Espíritu Santo (1590)(11) il était établi, en plus de la visite annuelle des directeurs, que tous les quatre mois le médecin et le chirurgien visiteraient, assistés de l'administrateur, tous les médicaments simples et composés en vérifiant s'ils sont fabriqués suivant les procédés et si quelque médicament est périmé, décidant à ce sujet ce qui leurs paraissait nécessaire. On indique, également, que l'apothicairerie pouvait être visitée chaque fois que l'administrateur le jugerait convenable et chaque fois qu'il le ferait il devait demander justification à l'apothicaire de ce qu'il avait reçu.

Les Ordonnances de l'Hôpital del Cardenal (1603) (12) établissent que l'apothicairerie doit être visitée par les médecins du centre et un apothicaire élu parmi les plus savants de la ville, deux fois par an, l'une en présence des directeurs et l'autre au mois de juillet en présence de l'administrateur, pour voir et examiner si les médicaments tant simples que composés présentaient la qualité convenable.

Dans les Ordonnances de l'Hôpital de San Cosme et San Damian, vulgairement dit de la Bubas (1512) (13) on ordonnait aux députés d'effectuer deux visites par mois à l'Hôpital, et à l'apothicairerie pour vérifier si elle disposait des eaux, sirops et de toute sorte de médicaments nécessaires et convenables pour la guérison et la santé des malades. Les dits députés, avec l'aide du médecin et du chirurgien, devaient examiner l'état dans lequel se trouvaient tous les articles et ordonner le remplacement de ceux qui étaient en mauvais état.

Dans les Constitutions de l'Hôpital de las Cinco Llagas ou de la Sangre (1624) (14) il y avait deux visites générales par an de la part des directeurs, de même que dans les autres hôpitaux, 
on inspectait l'apothicairerie. A part celles-ci, l'administrateur de l'hôpital, accompagné du médecin, devait la visiter deux fois par an, pendant les mois de septembre et mai, et chaque fois qu'il le jugerait nécessaire.

Bien que les Constitutions de l'Hôpital del Amor de Dios ne soient pas conservées des documents prouvent que des inspections étaient effectuées dans l'apothicairerie par l'administrateur du centre. Ainsi, lors de celle réalisée en 1760, y assistaient, le médecin et le chirurgien de l'hôpital et un maître pharmacien de la ville :

"En Sevilla en veinte y ocho de Mayo de mil setescientos y sesenta. El Sr. D. Joseph de Amat, Canónigo de la Santa Iglesia Patriarcal desta dicha ciudad y Administrador deste dicho Hospital del Amor de Dios, por ante mí el cura más antiguo y secretario de él, visitó la botica de dicho hospital, con asistencia del Sr. Dr. D. Francisco Gonzalez de León, Médico de Cámara de S. M., y titular deste dicho hospital, y Pedro Dominguez, Zirujano mayor de él, y hallándose presente D. Francisco Xavier de Atienza, Maestro Pharmacético desta ciudad, para efectos de examinar, provar y reconocer las Medicinas así simples como compuestas, como también la calidad y ley de los géneros $y$ drogas de dicha Botica, y de la suficiencia, surtimiento y provisión de ella, conforme a el gasto que regularmente se necesita para el alivio y curación de los enfermos..." (15).

En plus des inspections auxquelles étaient soumises ces apothicaireries de la part de l'hôpital lui même et des directeurs, elles l'étaient également par les Visiteurs nommés par la ville pour les inspections de toutes les apothicaireries incluses dans sa juridiction. Jusqu'en 1741, ces inspections étaient à la charge du Collège d'Apothicaires de Séville qui, par différents brevets du roi accordées par Carlos II, avait obtenu les titres de Visiteur (1685), Alguacil Majeur (1688) et Fiscal (1689) pour les visites des apothicaireries de Séville et de son Archevêché (16).

A partir de cette année, 1741, pendant laquelle on créa une subdélégation du Royal Protometicato dans Séville avec juridiction dans tout le territoire de l'Archevêché (17), les visites des apothicaireries incluses dans cette juridiction furent prises à sa charge. Cependant, cette subdélégation avait l'habitude de choisir comme Visiteur d'apothicairerie un apothicaire membre du Collège (18). A partir de 1780 , en conséquence de ce qui fut décidé par Carlos III le 13 avril pour que, au sein du Royal Protomedicato, les Facultés de Médecine, Chirurgie et Pharmacie se dirigent et gouvernent par elles mêmes, les inspections furent effectuées par le Visiteur Général d'Apothicaireries de la Ville de Séville et son Archevêché. Durant la visite à l'apothicairerie de l'Hôpital del Espíritu Santo en 1790 on spécifia, à ce sujet, qu'on l'effectuait "con arreglo al Real Despacho de los Señores Proctofarmacéuticos y juezes Alcaldes mayores examinadores perpetuos del Tribunal del Real Protomedicato de los Boticarios y cosas pertenecientes a la Facultad de Farmacia en todos los reinos y señorios de S.M., segun el folio tres de dicho Real Despacho dado en Madrid a seis de Noviembre de 1789. " (19). Lors de cette visite on cite également une Brevet du Roi, donnée par Felipe V, datée à Aranjuez du 23 juin 1743, et des traites émises par le nonce papal D. Enrique Enríquez à Madrid le 29 d'août 1747, en relation avec l'obligation qu'avaient les apothicaireries des couvents, collèges, maisons exemptes, oeuvres pieuses et hôpitaux, de se soumettre aux visites générales. 


\section{Personnel}

Dans tous les hôpitaux étudiés il y a un apothicaire responsable et un ou plusieurs garçons d'apothicairerie ou préparateur, qui parfois, quand il n'y avait pas d'apothicaire employé, agissaient en tant que tel. Ces garçons d'apothicairerie élus personnellement par les apothicaires, apprenaient leur métier à l'hôpital, où ils réalisaient des opérations. Les garçons d'apothicaireries, aussi bien que les apothicaires, devaient loger dans les hôpitaux, sans pouvoir s'absenter sans l'autorisation de l'Administrateur.

L'élection de l'apothicaire qui devait diriger l'apothicairerie de l'hôpital, était au choix de l'administrateur du Centre, bien que sa désignation dût être confirmée par le directeur. Le candidat à la place devait être célibataire, de bonnes mœurs et devait se soumettre à un examen effectué par le médecin titulaire en présence de l'administrateur et de quelques témoins, normalement l'infirmier en chef, les aides d'apothicairerie et le secrétaire, qui enregistrait l'acte. Les conditions exigées par les Constitutions des hôpitaux à la personne qui devait diriger l'apothicairerie, varient d'un centre à l'autre. Dans l'Hôpital del Cardenal on exige d'être très apte et expérimenté dans le métier, mais on ne mentionne pas qu'il devait être apothicaire diplômé. Cependant, à partir de 1663, la place d'apothicairerie semble acquérir un caractère plus professionnel dans le sens où l'on réserve sa provision aux apothicaires. Dans les hôpitaux del Espíritu Santo et de las Cinco Llagas, par contre, on exigeait un apothicaire expert et diplômé.

L'apothicaire se trouve toujours parmi les commissionnés mineurs ou non titulaires et son engagement varie d'un hôpital à l'autre. Dans les Hôpi- taux del Amor de Dios, del Cardenal et de las Cinco Llagas, on emploie durant tout le siècle des apothicaires qui exercent leurs fonctions dans l'apothicairerie de l'hôpital. Dans l'Hôpital de las Bubas et dans celui del Espíritu Santo, on combine cette formule avec l'établissement d'un contrat avec un apothicaire installé dans la ville, afin qu'il fournisse les médicaments nécessaires à la guérison des malades.

Dans l'Hôpital de las Bubas, depuis l'année 1643 jusqu'en 1709 , on emploie un apothicaire à raison de 500 Rs. pour chaque "Aguaje" (20) pour les drogues et le palissandre nécessaires, y compris le salaire de l'apothicaire. A partir de 1709, l'hôpital crée un contrat avec un apothicaire de la ville pour qu'il fournisse les médicaments nécessaires pour les six traitements à base de gaïac qui se font par an ; le prix fixé est de 1200 reals de billon (21). Depuis cette année et jusqu'à 1796 on continue ce système, bien qu'à partir de 1720 les contrats s'établissent en 1300 reals de billon.

Dans l'Hôpital del Espíritu Santo, les contrats avec un apothicaire de la ville s'établissent à partir de 1778 . Dans ce cas avec l'apothicaire D. José Espinosa, ayant une apothicairerie dans la rue de la Mar. Celui-ci occupa durant quelques mois de 1787 la place d'apothicaire de l'hôpital tout en fournissant au centre les drogues nécessaires à l'apothicairerie. Comme cela n'était pas conforme avec ce qui était établi et ayant peur d'être découvert, il fit part à l'administration de l'hôpital en janvier 1788 de son intention de cesser le service de l'apothicairerie, proposant l'établissement d'un contrat entre lui et l'hôpital par lequel, moyennant un montant pré-établi, il fournirait au centre les médicaments nécessaires.

L'administrateur, conseillé par le médecin de l'hôpital, D. Juan de 
Pereira, qui se prononça favorablement, demanda à l'Archevêque la licence nécessaire pour pouvoir formaliser le contrat dans les termes les plus équitables. La licence une fois obtenue, et après étude des frais imposés à l'apothicairerie - y compris ceux de l'apothicaire pour ses effets personnels, son alimentation et son salaire au cours des quinze dernières années, on fixa le montant du dit contrat à 500 ducats par an.

Ces contrats, par contre, pouvaient poser des problèmes, car le prix convenu ne coïncidait pas toujours avec celui des médicaments fournis par l'apothicaire, qui dans différentes occasions dépassait la quantité établie en raison d'une augmentation du nombre de malades. Ceci provoquait des conflits entre l'hôpital et l'apothicaire, qui parfois aboutissaient à un accord mais d'autres fois à un procès. Ainsi, l'apothicaire D. José Espinosa remit en septembre 1788 à l'administrateur de l'hôpital del Espíritu Santo la liste des frais journaliers des médicaments livrés et un résumé de tout ce qui avait été utilisé jusqu'à la fin juillet, demandant le versement du découvert qui apparaissait dans les comptes, ajoutant ce que l'on pouvait considérer convenable pour le travail de l'apothicaire et de son préparateur. L'administrateur et Espinosa convinrent de soumettre les comptes à l'examen de deux experts et d'accepter la décision de ces derniers. Les experts nommés furent $D$. Manuel de Lora y Bracamonte, Pharmacien et Examinateur du Royal Protomedicato dans la Sub-Délégation de Séville, et D. Jose Melgarejo, qui avait été nommé apothicaire de l'hôpital depuis le moment où Espinosa avait cessé l'approvisionnement de médicaments. Dans leur rapport les deux apothicaires indiquèrent que les dits comptes étaient corrects, vu la grande quantité d'articles utilisés pour les médicaments, mais que malgré cela et pour aider l'oeuvre pieuse à laquelle ils étaient destinés, ils avaient accordé de diminuer quelque peu la quantité mensuelle.

Mais ces conflits ne se résolvaient pas toujours à l'amiable, et ils se terminaient devant les tribunaux de justice, comme dans le cas de l'apothicaire D. Francisco Ruiz de Talabán, avec lequel l'I ôpital de las Bubas entama un procès en 1793 , car on considérait abusif le montant des 49 compositions fournies à l'Hôpital et qui atteignaient les 1,728 Rs. de billon et $15 \mathrm{mrs}$. L'Administrateur de l'hôpital indiquait que les malades admis cette année dans l'hôpital n'étaient que douze et que les années antérieures alors qu'on admettait de 50 à 60 malades, on ne payait à l'apothicaire que 1300 Rs. pour tous les médicaments qui étaient utilisés annuellement. D. Francisco Ruiz Talaban, de son côté, alléguait que, malgré la diminution du nombre de malades, la thérapeutique préconisée à l'hôpital avait changé et que les médicaments utilisés avaient un prix plus élevé.

L'Hôpital fit appel devant la Cour d'Appel de Séville, laquelle, en accord avec le Royal Protomedicato, nomma les pharmaciens D. José de Amores et D. Manuel de Lora y Bracamonte, Examinateurs du dit Tribunal à la ville de Séville, pour qu'ils effectuent l'examen et l'estimation des recettes en litige. Le premier d'entre eux, représentait l'hôpital, et le deuxième représentait D. Francisco Ruiz. Le rapport aboutit à la réduction de ladite quantité à 1027 Rs. de Billon (22).

Quand l'apothicaire entrait en possession de son poste, et aussi lorsqu'il terminait son exercice, on faisait un inventaire très détaillé du matériel, des instruments et des articles médicaux existant dans l'apothicairerie, dont on le tenait responsable. 


\section{Fonctions de l'apothicaire}

Les fonctions propres de l'apothicaire étaient semblables dans tous les hôpitaux. En plus de l'élaboration des médicaments, il devait s'arranger pour qu'il ne manque pas dans l'apothicairerie ni les simples ni les composés nécessaires. L'apothicaire achetait les articles lui-même et il devait présenter les comptes à l'administrateur. Une autre de ses fonctions consistait à accompagner le médecin lors des visites du matin et de l'après-midi qu'il faisait dans les infirmeries et écrire dans le cahier d'ordonnances les médicaments que celui-ci prescrivait à chaque malade, certains desquels devaient être administrés par l'apothicaire luimême. Les obligations des apothicaires dans les différents hôpitaux étudiés étaient les suivantes :

Dans l'Hôpital del Espíritu Santo l'apothicaire avait l'obligation d'accompagner le médecin et le chirurgien lorsque ceux-ci réalisaient leurs visites pour qu'il voit ce qu'ils prescrivaient et qu'il connaisse les malades à qui il fallait donner les médicaments, inscrivant dans un livre blanc chaque jour ceux qui étaient prescrits, indiquant le nom de chacun des malades et leur numéro de lit. Il devait apporter les médicaments que le médecin et le chirurgien prescrivaient à chaque malade dans un vase couvert de papier, où l'on écrirait le numéro de chaque lit pour que les médicaments ne soient pas changés ni confondus. Les purges et pilules devaient être administrées personnellement aux heures indiquées par le médecin et le chirurgien, prévoyant ce qui pouvait être nécessaire, suivant la qualité de la purge et la disposition du malade, pour qu'il l'avale et la retienne plus facilement. Si le nombre des malades était trop élevé, l'administrateur devait lui fournir de l'aide avec le conseil du médecin et du chirurgien.
Ceux-ci devaient, en plus, conseiller l'apothicaire sur la préparation des médicaments composés, sur les simples qui convenaient le mieux, vérifiant leur bonne qualité. A l'apothicaire, il lui était défendu de vendre et de donner quelque médicament que ce soit dedans et en dehors de l'hôpital sans l'ordonnance du médecin ou du chirurgien (23).

Dans l'Hôpital del Cardenal l'apothicaire était également obligé d'assister aux visites réalisées le matin et l'après-midi par les médecins et tenir un livre pour indiquer les médicaments prescrits, les purges et sirops, devant noter de sa propre main ce que le médecin prescrivait. En été les sirops devaient être distribués à cinq heures du matin et en hiver à sept heures. Les purges devaient être confectionnées la nuit d'avant pour pouvoir être données au malade à son heure (24).

Dans l'Hôpital de las Cinco Llagas ou de la Sangre, l'apothicaire devait également être présent lors de toutes les visites du médecin et tenir un livre où il devait écrire tous les médicaments, sirops et purges que le médecin prescrivait, devant tout accomplir ponctuellement. Les purges et pastilles devaient être prêtes la nuit d'avant dans les infirmeries ; les premières dans les vases et les deuxièmes dans leur papiers, avec le numéro des lits correspondants. Les sirops devaient être administrés personnellement aux heures indiquées par le médecin et également et ponctuellement tout ce qui était prescrit par le chirurgien. Il devait faire bien attention à ce que les purges et sirops soient donnés à leur heure, toujours au moment d'ouvrir les infirmeries avant la visite du médecin. Pour que les malades avalent les purges plus facilement on demandait à l'apothicaire de les leur donner avec des olives, avoir des chiffons imbibés de vinaigre et tout ce qui pourrait être utile. Il 
devait préparer tous les médicaments composés nécessaires, distiller toutes les eaux, prévoyant l'achat des drogues simples, pierres, ambre et musc lorsque l'occasion se présentait. Tout devait être acheté avec l'autorisation de l'administrateur et il était obligé de détenir tous les livres de consultation (25).

\section{Salaires}

Le salaire que recevaient les apothicaires pour leur travail dans l'apothicairerie de l'hôpital variait d'un centre à l'autre, dépendant peut-être du nombre des malades admis. Dans l'Hôpital del Amor de Dios l'apothicaire percevait 528 Rs. de billon par an, plus l'aides et rations - lesquels étaient perçus à raison de 132 Rs. par tiers (tous les quatre mois), ce qui était la façon de toucher l'argent de tous les fonctionnaires du centre. Ce salaire se maintint sans changement tout le long du siècle jusqu'en 1795 où il fut porté à 1600 Rs. -400 Rs. par tiers -. Le salaire établi à l'Hôpital del Cardenal fut, jusqu'en 1789, 396 Rs. par an, plus l'aides et rations. A partir de cette année là il fut porté à 540 Rs., plus 50 Rs. d'aides et $300 \mathrm{Rs}$. $20 \mathrm{mrs}$. pour la ration ( 890 Rs. de b. au total). Le salaire perçu par l'apothicaire à l'Hôpital del Espíritu Santo était de 396 Rs. par an, ce qui correspondait en réalité à deux ducats de salaire plus un pour les frais; une ration d'une demi-livre de viande de mouton, une demi-mesure de vin et un pain. Le salaire était payé divisé en trois tiers de 132 Rs. chacun. Ce salaire resta fixe jusqu'en 1788 où il fut porté à 1200 Rs. par an. A l'Hôpital de las Cinco Llagas ou de la Sangre, le salaire était de deux ducats par mois, 795 Rs. par an, plus la ration: une demi-livre de viande de mouton et une demi-livre de viande de bœuf, et les vendredis une demi-once de poisson frais et une demi-once de poisson salé ; une demimesure de vin et trois livres de pain. A partir de 1789 il augmenta à 840 Rs. par an.

Les aides sont des rétributions additionnelles qui se donnaient en fonction des pétitions faites par les apothicaires lorsque ceux-ci considéraient que le travail réalisé dans l'apothicairerie était bien supérieur à ce qu'ils touchaient comme salaire. Ces aides en arrivèrent à être touchées avec une telle périodicité, qu'elles en arrivèrent à être incluses aux honoraires provoquant ainsi de nouvelles demandes, et parfois de nouvelles concessions d'aides.

La basse rémunération des apothicaires dans les hôpitaux, en dessous de celles du médecin et du chirurgien, et parfois du saigneur, fut sans aucun doute une des raisons principales pour lesquelles ce poste de travail n'était pas très attrayant pour les professionnels les plus qualifiés. Le nombre élevé d'apothicaires qui passèrent par les apothicaireries des hôpitaux démontre, de toute évidence, qu'il s'agissait d'un lieu de passage pour beaucoup d'apothicaires qui obtenaient ainsi un salaire fixe, bien que pas très élevé, jusqu'à ce qu'ils puissent s'installer à leur compte.

On constate, de même, que bien que la Pharmacie fut déclarée en 1650 "Art scientifique en tout égal à la Médecine " par Felipe IV, les pharmaciens, qui n'avaient pas encore à l'époque d'études universitaires à faire et qui rendaient un service plutôt pratique et artisanal, avaient une considération sociale et culturelle inférieure à celle des médecins. Ce ne sera que bien après la première moitié du siècle que la profession pharmaceutique commencera à jouir d'une considération sociale plus en accord avec son acquis scientifique. 
Cette réalité restait patente dans les hôpitaux de Séville, où les médecins titulaires, la plupart d'entre eux membres de la Société Royale de Médecine (26) et autres Sciences, étaient favorables aux nouveaux courants européens et par conséquent appliquaient les traitements les plus innovateurs. Ceci, logiquement, exigeait de la part de l'apothicaire un meilleur niveau de préparation scientifique et professionnelle, ce qui se remarque notamment dans les contrats du dernier tiers du siècle concernant des apothicaires appartenant au monde pharmaceutique de Séville, car certains d'entre eux étaient membres titulaires de la Société Royale.

\section{Références :}

1. - Cf. Carmona Garcia, J. I. (1979) : El Sistema de Hospitalidad pública en la Sevilla del Antiguo Régimen. Séville ; COLlantes DE TERAN, F.(1886) : Memorias históricas de los Establecimientos de Caridad de Sevilla. Séville.

2. - Lopez Diaz, M. T. (1987) : Estudio Histórico-farmacéutico del Hospital del Amor de Dios de Sevilla (1655-1755). Séville.

3. - Martinez Garcia, C. (1993) : “El Hospital del Espíritu Santo de Sevilla (1587-1837) ». Séville. Thèse doctorale inédite.

4. - Herrera Davila, J. (1990) : "Visión histórico-sanitaria del Hospital de San Hermenegildo de Sevilla (1455-1837) ». Séville. Thèse doctorale inédite.

5. - MORENO TORAL, E. (1995) : "Estudio Social y Famacoterapéutico de la lepra : El Hospital de san Lázaro de Sevilla $(S . X I I I-X I X)$ ». Séville. Thèse doctorale inédite.

6. - Lopez Alonso, C. (1988) : Locura y Sociedad en Sevilla : Historia del Hospital de los Inocentes (1436 ?-1840). Séville.

7. - Lopez DiAz, M. T. (1994) : Aportación al estudio de la tuberculosis en la Sevilla ilustrada. Actes du XXXIII ${ }^{*}$ Congrès International d'Histoire de la Médecine. Grenade-Séville : $1-6$ septembre, 1992.

8. - Herrera Davila, J. (1990) : Op. cit., p. 325.

9. - GONZALEZ LARA, F. (1995) : "La Fammacia sevillana en el S: XVIII a través de los documentos notariales del Archivo de Protocolos de Sevilla (1775-1800) ". Séville, p. 126-127.

10. - MARTINEz GARCia, C. (1991) : Drogas importadas desde Nueva España (1686-1720). Estudio estadístico farmacoterapéutico. Séville.

11. - Archivo Histórico de la Diputación Provincial de Sevilla. (A.H.D.P.S.), Hospital del Espíritu Santo (H.E.S.), liasse 2-c ; MarTinez GarCiA, C. (1993) : Op. cit., p. 241-260.

12. - Biblioteca del Palacio Arzopispal de Sevilla (B.P.A.S.), Sig. 33/200 ; Biblioteca Capitular y Colombina de Sevilla (B.C.C.), Sig. 84-7-22.

13. - A.D.H.P.S. Hospital de las Bubas (H.B.), liasse 3.

14. - A.H.D.P.S. Hospital de las Cinco Llagas (H.C.L.), liasse 1.

15. - Lopez Diaz, M. T. (1987) : Op. cit., p. 80.

16. - Lopez Diaz, M. T. (1991) : Directorio de Boticarios. Introduction historique et analyse de l'œuvre de Francisco Ortega (Sevilla, 1625). Edit. facsimilé. Séville, p. XVI-XVIII.

17. - Cf. IbORRA, P. (1987) : Historia del Protomedicato en España (1477-1822). Valladolid.

18. - Lopez Diaz, M. T. ; Martinez Garcia, C. (1991) : Pleitos entre el Colegio de Boticarios de Sevilla y la ciudad de El Puerto de Santa Maria relativos a las inspecciones de boticas. Ars Pharmaceutica, XXXII (1-4) : 105-112,p. 11.

19. - Martinez Garcia, C. (1993) : Op. cit., p. 130.

20. - "Aguaje " était le nom populaire donné à la dénommée cure à base de guayaco, qui était administrée à l'hôpital. Les " aguajes " étaient administrés habituellement au printemps et en automne.

21. - A.H.D.P.S. H.B., liasse 26.

22. - A.H.D.P.S. H.B., liasse 39. 
23. - A.H.D.P.S. H.E.S. Estatutos y Costituciones que nos Don Rodrigo de Castro... mando y ordeno se guarden en el Hospital del Espíritu Santo de esta çiudad... (1590), liasse, 2-c.

24. - B.P.A.S. Ordenanzas fechas por los Sres. visitadores perpetuos deste Hospital de $S^{t}$ Hermenegildo para reformacion y govierno del, assi en lo espiritual como en lo temporal. Sig. 33/200.

25. - A.H.D.P.S. H.C.L. Ordenanzas del Hospital de la Cinco LLagas, Liasse, 1.

26. - Cf. Hermosilla Molina, A.(1970) : Cien años de Medicina Sevillana. 1970. 